\title{
Laboreal
}

Volume $8 \mathrm{~N}^{\circ} 1$ | 2012

Género, Atividades e Saúde

\section{Trabajadoras del sector de la limpieza : precariedad en el empleo, desigualdades temporales y división sexual del trabajo}

As trabalhadoras do sector das limpezas : precariedade no emprego, desigualdades temporais e divisão sexual do trabalho Les ouvrières du nettoyage : précarité d'emploi, inégalités de temps et division sexuelle du travail

The female workers of cleaning : precariousness of employment, inequalities of time and sexual division of labour

\section{Guy Lebeer y Esteban Martinez}

Traductor. Jose Alberto Riesco Sanz

\section{(2) OpenEdition}

\section{Journals}

Edición electrónica

URL: http://journals.openedition.org/laboreal/7018

DOI: $10.4000 /$ laboreal.7018

ISSN: 1646-5237

Editor

Universidade do Porto

Referencia electrónica

Guy Lebeer y Esteban Martinez, «Trabajadoras del sector de la limpieza : precariedad en el empleo, desigualdades temporales y división sexual del trabajo », Laboreal [En línea], Volume 8 №1 | 2012, Publicado el 01 julio 2012, consultado el 08 octubre 2019. URL : http://journals.openedition.org/ laboreal/7018; DOI : 10.4000/laboreal.7018

Este documento fue generado automáticamente el 8 octubre 2019.

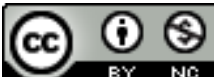

Laboreal está licenciado com uma Licença Creative Commons - Atribuição-NãoComercial 4.0 Internacional. 


\section{Trabajadoras del sector de la limpieza : precariedad en el empleo, desigualdades temporales y división sexual del trabajo}

As trabalhadoras do sector das limpezas : precariedade no emprego, desigualdades temporais e divisão sexual do trabalho

Les ouvrières du nettoyage : précarité d'emploi, inégalités de temps et division sexuelle du travail

The female workers of cleaning : precariousness of employment, inequalities of time and sexual division of labour

\section{Guy Lebeer y Esteban Martinez}

Tradución : Jose Alberto Riesco Sanz

\section{NOTA DEL EDITOR}

Manuscrito recibido en : Marzo/2012

Aceptado tras peritaje : Abril/2012

\section{Introducción}

1 En la mayoría de los países de la Unión Europea, el sector de la limpieza constituye una fuente importante de empleo para un segmento de población poco cualificada y mayoritariamente femenina. En Bélgica, casi dos de cada tres empleos en el sector están ocupados por mujeres. Sus condiciones penosas de trabajo son el resultado de una superposición de desregulaciones derivadas del recurso generalizado a la subcontratación y al trabajo a tiempo parcial (Puech, 2004, p. 152). La dinámica de la 
subcontratación y del incremento de la competencia entre las empresas del sector conduce, en efecto, a una espiral de reducción de costes y a un sometimiento permanente a las exigencias de los clientes que terminan por imponer distintas modalidades de intensificación del trabajo (Munar \& Lebeer, 2005). El continuo incremento del ritmo de trabajo y la adopción cada vez más frecuente de horarios desincronizados del resto de temporalidades sociales dominantes son dos de sus señales más tangibles. La confluencia de estas condiciones de trabajo y de salarios relativamente bajos han convertido al sector de la limpieza - industria en plena expansión que emplea en la actualidad a más de 3,6 millones de trabajadores en Europa (Scandella, 2010) - en fuente de un importante contingente de "trabajadores pobres" y con problemas de salud.

2 Este artículo presenta los resultados de un estudio encargado por el Centre de Formation du Nettoyage [Centro de Formación del Sector de la Limpieza], organismo gestionado de forma paritaria por los sindicatos y las organizaciones empresariales del sector belga de la limpieza. El estudio, titulado "Tiempo de trabajo, carga de trabajo y conciliación entre vida laboral y vida privada" (Lebeer, De Schampheleire \& Krzeslo, 2009), pretendía objetivar, en la medida de lo posible, las condiciones de trabajo de los asalariados del sector, unos trabajadores sujetos a la tendencia cada vez más habitual de los clientes a recurrir a la jornada partida en la organización del trabajo. La investigación pretendía servir como base científica para el desarrollo de una campaña de sensibilización destinada a los usuarios de los servicios de limpieza, tratando de llamar su atención sobre las consecuencias sociales de las condiciones impuestas por las empresas contratantes en materia de horarios al firmar el pliego de condiciones de los contratos.

3 A partir de los resultados de esta investigación cualitativa, efectuada en distintos centros de trabajo belgas y complementada mediante una contextualización estadística, el artículo trata de mostrar la importancia de las desigualdades de género dentro del sector de la limpieza, analizando al mismo tiempo su dinámica específica en el ámbito de los regímenes de trabajo, de las modalidades concretas de organización de tareas y de la gestión de la mano de obra. El artículo pretende asimismo poner en evidencia que el empleo de mujeres en el sector de la limpieza refleja los efectos de una doble segregación. En primer lugar, la segregación derivada de las condiciones específicas de subordinación que caracterizan al trabajo de limpieza efectuado -cada vez más- en el marco de relaciones de subcontratación. En segundo lugar, aquella que se desprendería de las tensiones, padecidas especialmente por las mujeres, entre horarios de trabajo no negociables y obligaciones de la vida privada.

\section{Metodología}

El estudio al que aquí nos referimos se desarrolló en dos fases. En una primera fase exploratoria, fueron consultadas las organizaciones sindicales y los principales empleadores del sector con el objetivo de conocer su valoración de las transformaciones registradas en la organización del trabajo y de sus efectos sobre las condiciones de vida y trabajo de los asalariados. En una segunda fase, se realizó una campaña de entrevistas a un total de 131 trabajadores y trabajadoras, contratados por cinco de las más importantes empresas belgas del sector y encargados de la limpieza en oficinas e instalaciones industriales en una decena de empresas del sector privado y de la administración pública [1]. 
5 La elección de los centros de trabajo contó con el acuerdo de los empleadores. Como investigadores, dicha elección trató de diversificar lo máximo posible las situaciones de trabajo consideradas tomando como referencia el tipo de actividad principal desarrollada por la empresa-cliente. De este modo, en el desarrollo de nuestra investigación hemos abordado, por ejemplo, desde un banco a la administración de una gran ciudad, pasando por una empresa del sector químico o una panadería industrial. En cada centro de trabajo hemos tratado de entrevistar al conjunto de los trabajadores del servicio de limpieza, si bien, lógicamente, al final sólo se han realizado aquellas entrevistas en las que los trabajadores han aceptado colaborar voluntariamente. Los trabajadores eran invitados a participar en entrevistas semi-directivas de una duración media de 30 minutos. Las entrevistas se llevaron a cabo en los propios centros de trabajo y durante la jornada laboral, apoyándonos en un guión estructurado en torno a las cuestiones siguientes : horarios de trabajo, descripción de las tareas desarrolladas y de la carga de trabajo, mecanismos de control, relaciones con los superiores y relaciones de trabajo, antigüedad y experiencias laborales previas, conciliación con la vida privada, críticas y propuestas de mejora.

6 El proceso de recogida y análisis de la información ha estado guiado, básicamente, por el deseo de evidenciar y comprender las estrategias de conciliación entre vida laboral y vida privada de estos trabajadores, así como los factores que eventualmente pudieran explicar las dificultades de dicha conciliación. La metodología aplicada no aspiraba, por lo tanto, a una producción sistemática de datos cuantificables que pudieran prestarse a un tratamiento de carácter estadístico. El hecho de que el trabajo de campo desarrollado no haya sido concebido con tal fin invalidaría, por lo tanto, toda pretensión de representatividad estadística.

7 La Tabla 1, que se presenta a continuación recoge, con seudónimo, algunas características de los centros de trabajo abordados en la investigación. La amplitud de la franja horaria de trabajo señala las horas durante las cuales la empresa de limpieza se encuentra presente en el centro de trabajo, por más que las tareas de limpieza propiamente dichas estén concentradas en secuencias más reducidas. Tal y como veremos, una de las principales características del sector es el recurso a la jornada de trabajo partida. Los turnos de trabajo varían considerablemente según los centros, e incluso dentro de un mismo centro. No obstante, en términos generales, suelen situarse por la mañana temprano (de $6: 00$ a $9: 00 / 10: 00$ ) y al final de la tarde (de $17: 00$ a 19: 00/22:00).

Tabla 1. Características de los centros de trabajo

\begin{tabular}{|l|l|l|l|}
\hline $\begin{array}{l}\text { Seudónimos de los } \\
\text { centros de trabajo }\end{array}$ & Tipo de trabajo & $\begin{array}{l}\text { Amplitud de la } \\
\text { franja horaria } \\
\text { de trabajo }\end{array}$ & $\begin{array}{l}\text { Número de } \\
\text { entrevistas }\end{array}$ \\
\hline $\begin{array}{l}\text { Banco "Citizen } \\
\text { Bank" }\end{array}$ & $\begin{array}{l}\text { Limpieza de los despachos, reciclado de } \\
\text { residuos }\end{array}$ & $6: 00-19: 00$ & 16 \\
\hline $\begin{array}{l}\text { Clínica "Paix Notre } \\
\text { Dame" }\end{array}$ & $\begin{array}{l}\text { Limpieza de las habitaciones de los } \\
\text { pacientes y de los despachos del personal } \\
\text { administrativo (las áreas médicas son } \\
\text { limpiadas por personal interno de la clínica) }\end{array}$ & $6: 00-18: 30$ & 11 \\
\hline
\end{tabular}




\begin{tabular}{|c|c|c|c|}
\hline $\begin{array}{l}\text { Escuela "Georges- } \\
\text { Perec" }\end{array}$ & $\begin{array}{l}\text { Limpieza de los locales de la escuela y del } \\
\text { comedor }\end{array}$ & $11: 00-20: 00$ & 4 \\
\hline $\begin{array}{l}\text { Empresa química } \\
\text { "Pfaff" }\end{array}$ & $\begin{array}{l}\text { Limpieza de los despachos, de los } \\
\text { laboratorios (zonas esterilizadas), gestión de } \\
\text { residuos. }\end{array}$ & $7: 00-20: 30$ & 12 \\
\hline $\begin{array}{lr}\text { Empresa } & \text { de } \\
\text { alimentación } & \text { "La } \\
\text { Gerbe d'Or" } & \end{array}$ & $\begin{array}{l}\text { Limpieza del espacio de administración y de } \\
\text { los talleres de producción }\end{array}$ & $00: 00-24: 00$ & 9 \\
\hline $\begin{array}{l}\text { Galería comercial } \\
\text { "Betapolis" }\end{array}$ & $\begin{array}{l}\text { Limpieza de todos los lugares de paso } \\
\text { (excepto los comercios) }\end{array}$ & $8: 00-20: 00$ & 2 \\
\hline $\begin{array}{l}\text { Instituto de } \\
\text { Investigación } \\
\text { Nuclear }\end{array}$ & $\begin{array}{l}\text { Limpieza del espacio de administración } \\
\text { (despachos y salas de reunión) y de los } \\
\text { laboratorios }\end{array}$ & $7: 30-15: 45$ & 4 \\
\hline $\begin{array}{l}\text { Tienda "Supply } \\
\text { Center" }\end{array}$ & $\begin{array}{l}\text { Limpieza de los pasillos y de los estantes, el } \\
\text { espacio de las cajas, el hall, así como los } \\
\text { locales de los empleados }\end{array}$ & $9: 00-22: 00$ & 4 \\
\hline $\begin{array}{l}\text { Empresa química } \\
\text { "Alfachemie" }\end{array}$ & $\begin{array}{l}\text { Limpieza de los despachos, de los } \\
\text { laboratorios y de los comedores }\end{array}$ & $7: 00-16: 00$ & 16 \\
\hline $\begin{array}{l}\text { Empresa siderúrgica } \\
\text { "Deltastahl" }\end{array}$ & $\begin{array}{l}\text { Limpieza de los despachos y de las salas de } \\
\text { maquinaria }\end{array}$ & $6: 00-19: 00$ & 17 \\
\hline $\begin{array}{l}\text { Administración } \\
\text { Pública de } \\
\text { Gammaville }\end{array}$ & $\begin{array}{l}\text { Limpieza de los despachos, de los espacios } \\
\text { de atención al público y de las salas del } \\
\text { museo }\end{array}$ & $6: 00-19: 00$ & 36 \\
\hline
\end{tabular}

\section{La subcontratación de las actividades de limpieza}

8 Según la European Federation of Cleaning Industries [Federación Europea del Sector de la Limpieza], entre 1989 y 2005 la tasa media de penetración del sector de limpieza europeo ha pasado del $43 \%$ al $61 \%(\mathrm{EFCI}, 2006)\left[^{2}\right]$. No resulta pues extraño que el número de empresas activas en el sector haya registrado un claro incremento, más aún si se tiene en cuenta que la inversión inicial requerida para entrar en el sector es relativamente pequeña. El trabajo de limpieza es, en consecuencia, una actividad ampliamente subcontratada pero que se lleva a cabo en la propia sede de la empresa-cliente. Estas características de la organización de trabajo determinarán el conjunto de las condiciones de trabajo. La externalización del servicio de limpieza, justificada con el argumento recurrente de la necesidad de focalización sobre la actividad principal ("core-business"), busca en realidad el establecimiento de un mayor control -véase una reducción- de los costes de la mano de obra. La competencia entre empresas en el momento de adjudicación de los contratos hace de la búsqueda de dicho objetivo un fenómeno estructural. La empresa que -tras proponer un precio por el servicio considerado por el cliente como menos costoso que su gestión interna- resulta 
adjudicataria del contrato por un período determinado, debe rentabilizar el trabajo llevado a cabo obteniendo un margen de beneficio. Dicho mecanismo, que se repetirá en las adjudicaciones posteriores, hace que "el sector se vea atrapado en una espiral de reducción de costes" (Munar \& Lebeer, 2005, p. 11) que terminará repercutiendo sobre las condiciones de trabajo por medio del incremento del ritmo de trabajo, de la fragmentación del empleo y de un riguroso ajuste de los tiempos de trabajo (cuando no, en los casos más extremos, mediante prácticas situadas al margen de la reglamentación vigente dentro del sector).

9 La inscripción del servicio de limpieza en una relación de subcontratación transforma asimismo la relación de empleo. La externalización supone la sustitución de una relación salarial clásica entre el empleador y el empleado por una relación triangular en la que interviene una empresa contratante. Los trabajadores del sector se encuentran así confrontados a una pluralidad de empleadores cuyas exigencias pueden resultar contradictorias entre sí. Dichos trabajadores se ven sometidos a una doble subordinación en la medida en que la empresa-cliente adquiere, de facto, un derecho de injerencia sobre la organización de trabajo a través del pliego de condiciones estipulado en el contrato, ejerciendo en ocasiones una autoridad directa sobre los asalariados de la empresa subcontratada. No obstante, dicha subordinación reforzada de los asalariados no siempre parece ser la consecuencia lógica de la dependencia económica de las pequeñas empresas subcontratadas con respecto a las empresas contratantes. En este sentido, por más que el sector de la limpieza siga albergando en su interior una multitud de pequeñas y medianas empresas, lo cierto es que la mayor parte del empleo se concentra en un número reducido de grandes empresas multinacionales que han extendido sus actividades a lo que hoy podríamos denominar "facility services", que incluirían, además del servicio de limpieza, labores de vigilancia, mantenimiento de edificios e instalaciones, restauración e incluso, en ocasiones, cesión temporal de trabajadores.

10 Así pues, las relaciones entre las empresas deberían más bien abordarse en términos de una connivencia comercial gracias a la cual la empresa-cliente logra reducir los costes del servicio de limpieza, aún a riesgo de privilegiar siempre a quien oferte el precio más bajo antes que a quien proponga un precio acorde con la búsqueda de calidad, la planificación y las condiciones de trabajo del servicio. La empresa subcontratada, por su parte, resultaría adjudicataria del servicio, teniendo además a su disposición la mano de obra -a la que tendrá que rentabilizar- previamente empleada en el centro de trabajo [3]. El conjunto de los trabajadores de los centros de trabajo considerados en la investigación se han visto afectados, con distinto grado de intensidad, por este tipo de procesos: incremento de los ritmos de trabajo, ajuste de los tiempos de trabajo, doble subordinación, fragmentación de los empleos...

\section{División sexuada del tiempo y del trabajo}

11 En el sector de la limpieza, el trabajo a tiempo parcial constituye una de las modalidades más habituales de organización del trabajo, incluso en aquellos países en los que dicha forma de empleo se encuentra, en términos generales, poco desarrollada. En la Unión Europea, dicho régimen de trabajo representa cerca del 70 \% del empleo del sector (EFCI, 2006). El trabajo a tiempo parcial es pues la estructura que posibilita la flexibilidad organizacional en dicha actividad, configurando así la opción 
habitualmente padecida por los asalariados. El trabajo a tiempo parcial se impone, fundamentalmente, como consecuencia de una organización del trabajo basada en la jornada partida que divide el tiempo de trabajo en dos bloques homogéneos (de día y de tarde) ; así como por la preferencia contrastada de los empleadores por un régimen de trabajo flexible que no conllevaría el pago de complementos salariales en caso de prestación de horas extraordinarias.

\subsection{Una división sexuada del tiempo de trabajo}

Si bien es cierto que, como consecuencia de la organización temporal de las prestaciones de servicio, el uso del trabajo a tiempo parcial se impone a todos los trabajadores, existe, no obstante, una división sexual del empleo que se refleja en la sobrerrepresentación de las mujeres dentro de este tipo de empleos $\mathrm{y}$, más concretamente, en aquellos caracterizados por prestaciones de servicio de menor duración (Cf. Tabla 2 situada más abajo). Por regla general, los hombres ocupan más a menudo empleos a tiempo completo o a tiempo parcial pero dotados de una duración mayor de la prestación laboral, mientras que las mujeres, empleadas la mayoría de las veces a tiempo parcial, ven cómo se les asignan los trabajos de menor duración. Así pues, más que el régimen de trabajo contractual, es el reparto del empleo en función del número de horas efectivamente trabajadas el principal indicador de las desigualdades de género. En Bélgica, mientras que en el sector de la limpieza la duración normal de la jornada de trabajo semanal a tiempo completo es de 37 horas, podemos constatar que sólo el $13 \%$ de las mujeres trabajan más de 30 horas por semana (frente al $62 \%$ en el caso de los hombres). Al mismo tiempo, un $28 \%$ de mujeres estarían ocupadas menos de 15 horas por semana, es decir, una media de menos de 3 horas por día (frente al $14 \%$ de los hombres). En términos generales, la duración del tiempo de trabajo real de las mujeres en el sector es de media jornada o menos, mientras que la mayoría de los hombres mantiene prestaciones de servicio de una duración más cercana a la jornada a tiempo completo.

Tabla 2. Duración del trabajo en el sector de la limpieza según sexo

\begin{tabular}{|l|l|l|}
\hline & Hombres & Mujeres \\
\hline Menos de 15 horas a la semana (menos de 3 horas por día) & 14,4 & 28,4 \\
\hline Entre 15 y 30 horas a la semana (entre 3 y 6 horas por día) & 23,4 & 58,1 \\
\hline Más de 30 horas a la semana (más de 6 horas por día) & 62,2 & 13,4 \\
\hline & 100,0 & 100,0 \\
\hline
\end{tabular}

Fuente : Memoclean (2005), base de datos de la Union Belge des Entreprises de Nettoyage et de Désinfection (UGBN) [Unión Belga de Empresas del Sector de la Limpieza y la Desinfección].

El análisis efectuado de los centros de trabajo muestra claramente que dicha diferenciación en el empleo es el resultado de la combinación de una división sexual del trabajo y de exigencias de disponibilidad temporal para el trabajo poco compatibles con las obligaciones familiares. Una exigencia que, como veremos, se articulará con una de 
las principales características del sector : la jornada de trabajo partida y con horarios desincronizados.

\subsection{Una división sexuada de las tareas}

14 Los hombres se encuentran mayoritariamente presentes en los subsectores de la limpieza industrial, la recogida de basuras y la limpieza de cristales, es decir, en aquellas tareas y funciones asociadas a salarios por hora superiores a los existentes en el sector de la limpieza de oficinas y de espacios colectivos equiparados. Se trata, además, de las tareas y funciones que, de acuerdo con los clientes, pueden ser llevadas a cabo de manera continuada durante los horarios normales y colectivos de la jornada de trabajo. Por otro lado, la especialización sexual del trabajo quedaría igualmente de manifiesto en el propio marco de la limpieza de oficinas, que representa cerca del $55 \%$ del volumen de negocio del sector y ocupa a la mayor parte de los empleados. En esta actividad mayoritaria, los hombres se encuentran habitualmente adscritos a la limpieza "pesada" que requiere el uso de maquinaria, así como a los trabajos que conllevan - o, al menos, permiten - una presencia de los empleados de carácter más permanente.

Desde el punto de vista de la clasificación sectorial -ampliamente utilizada por las empresas- de las funciones efectuadas en la limpieza de oficinas, hombres y mujeres se encuentran, en términos generales, en un plano de igualdad. No obstante, cuando se observa más de cerca la distribución de tareas en los centros de trabajo investigados, vemos que, en realidad, hombres y mujeres no son intercambiables. Así, por ejemplo, en muchos centros de trabajo en los que hombres y mujeres tienen asignadas tareas de limpieza, se presupone que, en caso de necesidad, los hombres echarán una mano o completarán su horario en alguna de las funciones de carácter cuasi-logístico que acompañan a las tareas de limpieza: evacuación de residuos, desplazamiento de contenedores, pequeñas reparaciones. A este tipo de tareas habría que añadir otras como el manejo de maquinaria (abrillantadoras, fregadoras, limpieza a presión, etc.). En la mayoría de los casos, los equipos de limpieza "especial", es decir, las operaciones especializadas y puntuales de mantenimiento, son masculinas. El caso de un empleado que trabajaba en las instalaciones de Citizen Bank resulta representativo del modelo de empleo masculino vigente en la limpieza de oficinas. Este trabajador limpiaba cada mañana la acera situada enfrente del edificio. En el interior pasaba la fregadora, la abrillantadora y limpiaba la moqueta. Se encargaba igualmente de la logística y descarga del material sanitario (toallas, papel higiénico, productos de limpieza) en el local previsto a tal efecto, es decir, de las tareas denominadas "logísticas". Asimismo, efectuaba actividades de limpieza clásicas pero sólo para cubrir bajas eventuales. Dicho trabajador estaba contratado a tiempo completo y siempre con jornada de trabajo partida.

Por más que se afirme formalmente que las tareas de limpieza se asignan indistintamente a hombres y a mujeres, basta con mirar con mayor detenimiento, más allá de descripciones evasivas del tipo "por las mañanas limpio los despachos", para que emerjan diferencias sutiles. Así, por ejemplo, en este mismo centro de trabajo al que nos estamos refiriendo, es muy frecuente que las empleadas sean asignadas exclusivamente a la limpieza de los despachos, provistas de un carrito y una bayeta. Del mismo modo, en la clínica Paix Notre Dame, son las mujeres quienes se encargan de las habitaciones y de las oficinas del personal administrativo. El contacto con los pacientes 
y sus familias es igualmente responsabilidad exclusiva de ellas. Los hombres, por su parte, se encargan de los pasillos y de los sanitarios. En la empresa química Pfaff, las tareas son también distintas en un caso y otro : los hombres se encargan de la gestión de los residuos, del transporte de los contenedores, del reciclado y de la conducción de carretillas elevadoras, mientras que las mujeres se encargan de la limpieza de los laboratorios.

En definitiva, en la limpieza convencional de oficinas, las disparidades salariales existentes entre hombres y mujeres se explican más por las diferencias en la duración efectiva del trabajo que por el lugar formalmente ocupado en la jerarquía salarial. A pesar de que hombres y mujeres anhelan por igual trabajar más, los horarios prolongados y los complementos de horas son concedidos prioritariamente a los hombres. Esta división sexual del empleo, que atribuye a los hombres una duración de la jornada de trabajo más próxima al tiempo completo, se suma a una distribución sexualmente diferenciada de tareas y a una representación particular de los roles de género en la familia y en el trabajo.

Siguiendo Angeloff (1999: 61), podríamos, en efecto, analizar la división del trabajo vigente en los centros investigados a partir de las distintas lógicas presentes en la distribución del trabajo en un contexto generalizado de escasez de los empleos a tiempo completo. Al igual que ella, en nuestra investigación también hemos podido constatar la existencia, por un lado, de una lógica de género según la cual el jefe de equipo, por lo general un hombre, tendería a privilegiar a los varones (incrementando su tiempo de trabajo hasta asimilarlo a una jornada a tiempo completo) y delegaría en las mujeres el tiempo de trabajo parcial (aún cuando éstas pudieran contar con una mayor antigüedad). Por otro lado, nos encontraríamos ante una lógica típica de la inmigración por medio de la cual se reforzaría la idea de que es el varón el cabeza de familia y que, desde este punto de vista, debe ser privilegiado en el reparto de las horas de trabajo, por más que muchas de estas mujeres se encuentran, en realidad, en una situación de monoparentalidad [4]. A estas lógicas podríamos añadir, finalmente, una lógica "étnica" que tendería a favorecer al propio clan, a los próximos, a la familia entendida en un sentido amplio.

\subsection{Jornadas partidas de trabajo y horarios desincronizados}

En la mayoría de los centros - incluidos los abordados en este estudio - el trabajo está organizado en jornada partida, quedando así concentrado en torno a dos franjas horarias : al comienzo de la mañana y al final de la tarde, sin que esto suponga para el trabajador la percepción de complemento salarial alguno. El trabajo nocturno propiamente dicho, por el contrario, es poco frecuente. En los países de la Unión Europea, como media, tan sólo el $25 \%$ de la actividad de limpieza efectuada por empresas externas se realiza durante las horas convencionales de la jornada de trabajo (EFCI, 2006). En un mercado fuertemente competitivo, las empresas-cliente pueden así imponer a las empresas subcontratadas horarios incómodos que garanticen que su personal no se verá importunado por el trabajo de limpieza.

No obstante, es evidente que dicha organización temporal no tiene el carácter ineluctable que, en ocasiones, se le atribuye. Este tipo de organización sería más bien el resultado del acuerdo implícito existente entre empresas-cliente y empleadores para reducir el coste del servicio de limpieza mediante una intensificación del trabajo a 
través de la supresión de los tiempos muertos, las pausas, las interrupciones y perturbaciones, así como mediante la configuración del tiempo de trabajo remunerado por medio de la movilización del trabajo según bloques horarios potencialmente acoplables entre sí. A poco que se observe con mayor detenimiento, veremos que la naturaleza de la propia actividad no basta para justificar este modo de organización. Así, por ejemplo, en Suecia, aunque es posible que se trate de una excepción nacional, la organización del sector de la limpieza en torno a la jornada de trabajo convencional se ha convertido en algo habitual y representa el $70 \%$ de la actividad (EFCI, 2006). Podemos constatar igualmente que en los centros de trabajo -como el metro de Bruselas- donde existe una fuerte presencia masculina, el grueso del trabajo de limpieza se desarrolla a lo largo de toda la mañana, en horas con una fuerte afluencia de público (Martínez, 2010).

21 Esta organización contingente del trabajo es una de las principales causas de la renuncia al trabajo a tiempo completo, sobre todo entre las mujeres, que siguen asumiendo la mayor parte de las responsabilidades domésticas y familiares. A diferencia de otras actividades caracterizadas por horarios atípicos e irregulares -como la restauración rápida que emplea sobretodo a jóvenes y estudiantes (Brochiez, 2001) o el sector fundamentalmente masculino de la seguridad privada (Cortese, Martínez \& Munar, 2003)- el sector de la limpieza emplea mayoritariamente a mujeres susceptibles de tener hijos a su cargo : casi el $60 \%$ de las empleadas pertenecen a franjas de edad intermedias (25-45 años). De modo que la jornada partida plantea, cuanto menos, el problema de la duración de la jornada de trabajo y de la desincronización de las franjas horarias de trabajo con respecto a las temporalidades sociales dominantes, así como la cuestión de los tiempos intermedios "captados" por el trabajo pero no remunerados. Dicho de otro modo, la jornada partida pone sobre la mesa el problema del desajuste que puede existir entre el tiempo real consagrado a la actividad profesional y el tiempo de trabajo propiamente remunerado. En un escenario en el que las trabajadoras se ven obligadas a renunciar a una $u$ otra de las franjas horarias en las que se divide la jornada de trabajo, no resulta sorprendente que la jornada partida constituya una causa importante de abandono para muchas de ellas.

\section{La exigencia de disponibilidad para el trabajo}

El recurso al trabajo a tiempo parcial, la jornada partida y los horarios desincronizados a la hora de organizar el trabajo son considerados por los empleadores -así como por algunos trabajadores- como una realidad ineludible dentro del sector, un atributo específico de la propia actividad de limpieza. La articulación de estas tres características de la organización temporal de los trabajos sitúa a los asalariados del sector de la limpieza en tensión con respecto a los ritmos socio-temporales dominantes que definen el reparto entre tiempos personales y tiempos profesionales para la mayoría de la población. La aceptación de este régimen de trabajo es una condición para su contratación y pervivencia en el sector. El rechazo de dicho régimen de trabajo, pese a ser causa habitual de queja entre los asalariados de nuestro estudio, resulta pues impensable. En base a su valoración del trabajo de limpieza, estos asalariados están persuadidos de que ninguna de sus tareas requiere un número suficiente de horas que pudiera justificar su contratación a tiempo completo y en un único emplazamiento. Si no hay horas suficientes es, precisamente, porque resulta supuestamente obvio que las 
labores de limpieza no pueden efectuarse sino cuando los asalariados de la empresacliente se encuentran ausentes (antes o después). Los testimonios recogidos confirman esta idea :

\footnotetext{
"Siempre, la limpieza siempre es un servicio con jornada de trabajo partida, ¿cómo vas a hacer por la mañana o por la tarde para tener un tiempo completo ?" (Trabajadora, Galería Betapolis)

"¿El mejor horario ? De $8: 00$ hasta después de comer, de una vez. Pero eso es imposible: los empleados están trabajando en los despachos durante la jornada. Si voy a las 11:00 la gente estará allí. No se puede (...). En primer lugar, a las $15: 00$, hacer las papeleras te lleva una hora. Los empleados están ahí. Se van a las $16: 00$. Donde no hay nadie limpiamos las oficinas. Después los baños cuando está todo tranquilo. Más tarde los despachos que no han podido hacerse antes porque había aún gente" (Trabajadora, Citizen Bank)
}

La presencia de los asalariados de la empresa-cliente es percibida como un elemento perturbador que ralentiza el ritmo de trabajo : es necesario esperar a que todo esté tranquilo para cumplir con los tiempos previstos para cada una de las tareas. En consecuencia, la posibilidad de estar ocupado con un horario, aunque sea variable, durante la jornada de trabajo convencional es considerada como una gran suerte, sobre todo cuando se han conocido previamente los horarios desincronizados.

\subsection{Una definición ambigua del tiempo de trabajo}

La tolerancia de los trabajadores ante las malas condiciones de trabajo se explica en gran medida por el hecho de que todos buscan ampliar el número de horas de trabajo, es decir, por la reivindicación -casi siempre ante los jefes de equipo- de un incremento de la duración del trabajo reconocida contractualmente, por la prestación de horas extra o por la acumulación de distintos trabajos. En este sentido, la disponibilidad temporal que ofrecen los asalariados responde a una lógica de subsistencia o, dicho en otros términos, a una necesidad de carácter principalmente económico (Thoemmes, 2000). La duración del trabajo se encuentra normalmente vinculada a una de las dos franjas horarias de la jornada partida (por ejemplo, 3 horas por la mañana ó 3 horas por la tarde, lo que equivaldría a 15 horas por semana). Se trata pues, necesariamente, de un empleo a tiempo parcial. En ocasiones, el asalariado logra "ganar horas" utilizando diversas estratagemas como, por ejemplo, añadir otro turno de trabajo en el mismo centro o recurrir a distintos empleadores. En este sentido, es necesario señalar que hombres y mujeres no se encuentran en pie de igualdad a la hora de buscar un horario de trabajo que garantice unos ingresos razonables $y$, al mismo tiempo, un equilibrio siempre frágil entre empleo y vida familiar.

La pluriactividad, es decir, la ocupación de varios empleos por parte de una misma persona, es una situación frecuente en el sector de la limpieza. Cerca del $14 \%$ de los asalariados del sector ocupan varios empleos simultáneamente o a lo largo del mismo trimestre, mientras que la tasa de pluriactividad media para el conjunto de los asalariados es del $7 \%$ (Cf. Tabla 3 situada más abajo). Además, en el sector de la limpieza, la pluriactividad hace referencia a un cúmulo de empleos asalariados (ejercidos únicamente en el sector de la limpieza), cuando, en términos generales, dicha tasa suele reflejar la vinculación de un empleo asalariado con un trabajo por cuenta propia. 
Tabla 3. La pluriactividad de los asalariados

\begin{tabular}{|l|l|l|l|l|l|l|}
\hline \multirow{2}{*}{} & \multicolumn{4}{|l|}{ Total de los asalariados (\%) } & \multicolumn{3}{l|}{ Sector de la limpieza (\%) } \\
\cline { 2 - 8 } & Hombres & Mujeres & Total & Hombres & Mujeres & Total \\
\hline En un empleo asalariado & 92,4 & 94,4 & 93,3 & 86,8 & 85,3 & 85,8 \\
\hline En varios empleos asalariados & 2,3 & 3,3 & 2,7 & 10,2 & 13,5 & 12,4 \\
\hline Cuenta propia y asalariado & 5,3 & 2,4 & 4,0 & 3,0 & 1,3 & 1,9 \\
\hline Total & 100,0 & 100,0 & 100,0 & 100,0 & 100,0 & 100,0 \\
\hline
\end{tabular}

Fuente : Banque Carrefour de la Sécurité Sociale, Bélgica, 2008. Elaboración propia a partir de una muestra de la población asalariada.

Estos datos concuerdan con las declaraciones que, en relación a la pluriactividad, hemos recogido en nuestro trabajo de campo. Así, por ejemplo, un trabajador ocupado desde las 6:00 hasta las 8:00 en la clínica Paix Notre Dame señalaba haberse compuesto un horario de trabajo a tiempo parcial añadiendo otras dos franjas horarias obtenidas de dos empleadores diferentes en dos centros de trabajo distintos. Así, además de su trabajo inicial, este trabajador estaba empleado al mismo tiempo en otro hospital de $18: 00$ a $21: 00$ durante la semana y de $14: 00$ a $20: 00$ los sábados. Con estos tres módulos temporales lograba estar ocupado 6 días a la semana con un total de 30 horas. Es el caso también de una limpiadora empleada en la tienda Supply Center que trabajaba-siempre a tiempo parcial- para tres empleadores distintos. Por la noche, en su primer contrato a tiempo parcial, trabajaba de $19: 00$ a $22: 00$. Los miércoles por la mañana, de $8: 30$ a 10 : 30, se desplaza al centro comercial Galería Betapolis, donde tenía su segundo contrato a tiempo parcial. Finalmente, los viernes por la mañana, con su tercer contrato a tiempo parcial, trabaja de $8: 30$ a $12: 30$ en una empresa industrial. Al final, no computaba más que 21 horas de trabajo semanales.

Estas situaciones de pluriactividad se pueden constatar también en los casos en los que el asalariado, pese a contar con un único empleador, se encuentra ocupado en varios centros diferentes. En este tipo de situaciones no resulta extraño que se firmen varios contratos de trabajo, de manera que los preavisos en caso de despido puedan ser notificados de forma independiente unos de otros. El propio centro de trabajo es el que determina el volumen global de trabajo y su localización diaria y semanal (incluso anual en el caso de determinadas tareas), sin embargo, la duración del trabajo estipulada por contrato se encuentra moldeada por las distintas secuencias de trabajo existentes. Por ejemplo, en el centro escolar Georges-Perec el servicio de comedor da lugar a un primer contrato de trabajo y la limpieza de las aulas a un segundo contrato. Cuando los trabajadores muestran su preferencia por un contrato a tiempo parcial lo hacen, a menudo, porque cuentan con otro empleo en otra empresa. La duración del trabajo y, por lo tanto, la remuneración, depende de la acumulación de distintas franjas temporales definidas por contrato. No pudiendo obtener un contrato a tiempo completo, o ante la imposibilidad de hacerse cargo del horario propuesto, los trabajadores tratan de incrementar su tiempo de trabajo combinando, siempre que sea 
factible, distintas franjas horarias de trabajo que sean compatibles con las obligaciones de la vida privada. Sin embargo, esta situación de pluriactividad plantea, por otro lado, el problema de la sincronización de los distintos fragmentos del tiempo de trabajo. Y es que, cada jefe de equipo, cada empleador dicta sus exigencias temporales sin tomar en consideración la situación personal del asalariado.

Angeloff (1999) describe perfectamente la ambivalencia presente en esta búsqueda de una duración de trabajo próxima al tiempo completo: "trabajar a tiempo parcial supone también recibir un salario parcial. Esto implica estar preparado para incrementar el salario y el tiempo de trabajo siempre que sea posible, cayendo de este modo en la espiral del trabajo a tiempo parcial flexible. La flexibilidad, por su parte, alimenta la esperanza de poder 'hacer más horas' y, por lo tanto, de incrementar, aunque sea mínimamente, un salario irrisorio" (p. 61). En las entrevistas efectuadas en nuestro trabajo de campo resulta a menudo complicado recabar una información precisa sobre la duración real del tiempo de trabajo. De hecho, los asalariados se encuentran a menudo contratados con un contrato a tiempo parcial que cubre únicamente una parte de la jornada de trabajo. El resto de la jornada se compone de horas extraordinarias que no implican una modificación del contrato de trabajo en tanto en cuanto dichas horas extra son consideradas de carácter provisional (cobertura de bajas, tareas ocasionales, etc.).

La medida del tiempo de trabajo estipulado por contrato puede resultar, además, un tanto confusa para los asalariados que trabajan en distintos centros o para diferentes empleadores. En ocasiones se considera "tiempo completo" a un horario que no alcanza las 37 horas semanales pero cuya duración -calculada tras sumar todas las secuencias de trabajo diarias o semanales- se aproxima. Es el caso, por ejemplo, de una mujer empleada en la sede de Citizen Bank que afirmaba trabajar a tiempo completo pero que, en realidad, se trataba de la suma de dos trabajos a tiempo parcial (uno de mañana, de 6:00 a 8:00 en la sede del banco; y otro de tarde, de 18:00 a 21:00 en otro emplazamiento) formalizados en dos contratos de trabajo diferentes ante un mismo empleador. Otra trabajadora, jefa de equipo en la tienda Supply Center, afirmaba contar también con dos contratos que juntos equivaldrían a un tiempo completo. No obstante, esta trabajadora contaba con un contrato indefinido para las horas que realizaba al final de la tarde (de 19:00 a 22:00) y un contrato de interinidad para el trabajo efectuado durante el día (de $14: 00$ a 18 :00). En la mayoría de los casos, la duración de la jornada de trabajo es amplia -yendo, en ocasiones, de $6: 00$ de la mañana a $22: 00$ de la noche, sin contar los tiempos de desplazamiento- mientras que las horas de trabajo remuneradas dentro de esta franja horaria difícilmente equivaldrían a un trabajo a tiempo completo. La acumulación de horas de trabajo o de empleos es frecuente, convirtiéndose en norma habitual dentro del sector :

"Antes aún era posible lograr el equivalente a un tiempo completo con dos trabajos de 4 horas, pero los clientes prefieren ahora prestaciones de servicio de una duración cada vez más concentrada, aunque eso suponga tener que movilizar un mayor número de trabajadores en el centro" (Jefa de zona)

Se multiplican así los pequeños trabajos de unas pocas horas de duración, como ejemplifica uno de los centros de trabajo abordados en nuestro estudio. En dicho centro las empleadas trabajaban de $5: 00$ a $7: 15$, retomando posteriormente su trabajo de 17 : 00 a $20: 15$. Es decir, un trabajo de 5 horas y 30 minutos con una disponibilidad de 9 horas. Las asalariadas del ejemplo dispondrían formalmente de 7 horas de tiempo libre 
durante la jornada pero sus noches serían, en el mejor de los casos, de 6 horas de duración. Con un modelo semejante resulta difícil imaginar un mecanismo para impedir que los horarios de trabajo invadan los tiempos reservados a la vida familiar y personal.

\subsection{Tiempos de trabajo desbordados}

Durante nuestra investigación hemos constatado que, dentro del sector de la limpieza, el recurso a la jornada de trabajo partida está en el origen de buena parte de las dificultades registradas para conciliar vida laboral y familiar. De hecho, la organización del trabajo por medio de la jornada partida conlleva también el recurso a los horarios desincronizados. El objetivo es diferenciar los tiempos de trabajo de las limpiadoras del de los clientes, que se encuentran casi siempre presentes en los centros de trabajo durante la jornada laboral estándar. En el trabajo de oficina, lo habitual es que los empleados comiencen su jornada entre las $8: 00$ y las 9:00 de la mañana y la terminen hacia las $17: 00$ ó las $18: 00$ como máximo. Los horarios de las limpiadoras deben pues ubicarse entre las $6: 00$ y las $9: 00$ de la mañana y terminar como máximo a las $22: 00$ de la noche, ya que más allá de dicha franja horaria entraría en vigor el trabajo nocturno, que conllevaría complementos salariales. La amplitud de la jornada es de 16 horas, pero los turnos de limpieza deben evitar, en la medida de lo posible, las horas en las que los empleados de la empresa-cliente se encuentran presentes. Dicho de otro modo, en lo que a los horarios de limpieza se refiere no se tiene en cuenta el propio ritmo de la vida de las personas, las obligaciones de la vida familiar, personal y social o las tareas a realizar durante el período de "no trabajo" -difícilmente asimilable a un tiempo de "descanso"disponible entre dos turnos de trabajo.

Los horarios de trabajo fragmentados entre diferentes secuencias de trabajo, distintos centros de trabajo y empleadores variados sobrecargan el tiempo consagrado por los asalariados a su actividad profesional. La jornada de trabajo partida conlleva una multiplicación de las idas y venidas durante una misma jornada, los tiempos parciales de corta duración exigen desplazamientos para llegar al centro de trabajo de una duración similar a los de la propia prestación de servicio, como ponía en evidencia la absurda situación de dos limpiadoras enviadas a una zona industrial para efectuar un trabajo de dos horas de duración y cuyo desplazamiento implicaba un tiempo de una hora y media. El coste es aún mayor cuando los centros de trabajo se encuentran alejados unos de otros o si escasea (o no hay) el transporte público. Fuera de los centros urbanos, la aplicación de horarios desincronizados dificulta aún más la situación en la medida en que la frecuencia del transporte público se organiza en función de horarios estándar que no se corresponden con las prácticas del sector de la limpieza.

El coste del desplazamiento afecta, principalmente, a las mujeres ya que, en términos generales, suele ser su compañero varón quien conduce el coche familiar y porque su tiempo se encuentra más ligado a las obligaciones familiares que el de los varones. Las dificultades para el desplazamiento, en un contexto en el que se requiere ser móvil y aceptar múltiples trabajos, se convierten así en un obstáculo para el empleo, reforzando su dependencia con respecto al cónyuge. El uso del tiempo de esta trabajadora empleada en la Galería Betapolis resulta revelador a este respecto :

"Comenzaba por la mañana, a las $6: 00$. Bueno, mi marido me llevaba en coche. A las $10: 00$ había terminado y tenía que tomar el autobús (...). Llegaba 
al tranvía, lo tomaba y después me tocaba hacer trasbordo. A veces no tenía la correspondencia inmediatamente, la perdía y llegaba a casa a las $12: 45$ ó las $13: 00$, todo para hacer 3 horas de trabajo. Tenía el tiempo justo para ir a buscar a los niños, darles la comida y volver a llevarles a la escuela. Luego tomaba de nuevo el tranvía para volver al trabajo. Terminaba a las $19: 00$. Mi marido venía a buscarme. De 19:00 a $22: 00$ tenía aún otro trabajo. Para lograr hacer siete horas y media terminaba a las $22: 00$ " (Trabajadora, Galería Betapolis). casos parecen viables ciertos apaños. Es el caso, por ejemplo, de una limpiadora de la escuela Georges-Perec que afirmaba estar satisfecha: trabajaba de 11:30 a 13:30 y después retomaba su trabajo a las 16:30 en otro centro. Esta trabajadora aprovechaba la interrupción de la jornada de trabajo para preparar la comida. No obstante, es cierto que, en términos generales, el uso del tiempo libre disponible entre el turno de mañana y el turno de tarde varía entre hombres y mujeres. Hay hombres que dicen aprovechar ese tiempo para descansar o para ver a sus hijos los días que no tienen escuela y hacerles la comida al mediodía; otros aprovechan para hacer gestiones administrativas. Todas las mujeres, sin embargo, afirman aprovechar para preparar la cena, limpiar la casa y atender las obligaciones familiares. Tal y como ha demostrado Barrère-Maurisson $(2000: 310)$ a partir de sus investigaciones sobre el uso del tiempo, las mujeres ocupadas a tiempo parcial consagran la mayor parte del "tiempo liberado" a las responsabilidades familiares y muy poco al ocio o al cuidado personal. Así pues, aunque el tiempo disponible entre dos turnos de trabajo pueda compensar los inconvenientes de los horarios desincronizados, no logra revertir la división sexual de tareas existente $\mathrm{y}$, en el caso de las mujeres, es evidente que dicho tiempo no representa para ellas un tiempo libre y de descanso.

"Me iba a trabajar. Mientras que están en la escuela no hay ningún problema, pero luego, cuando están en casa, hay que estar llamando cada cinco el trabajo y hay que pedirle el favor a alguien... Francamente, era un horror" (Trabajadora, Galería Betapolis).

"Cuando llego a casa son las $11: 00$ de la mañana. Es el momento de hacer la comida... Luego, si tengo que hacer algunas compras, dos o tres cosas, enseguida me dan las $15: 00$. Y digo 'uf'. Me siento ya cansada por haberme levantado a las $5: 00$ de la mañana y debo volver a trabajar. Vuelvo a casa por la noche, hacia las $20: 00$ ó las $21: 00$, depende. Para cuando me voy a dormir ya son las $23: 00$, medianoche y me tengo que levantar a las $5: 00$. A la larga estás permanentemente cansada" (Trabajadora, Pfaff).

Los testimonios recogidos durante el trabajo de campo destacan el hecho de que, más allá de su impacto físico o psicológico, la jornada de trabajo partida supone para los 
asalariados un nivel de compromiso profesional desproporcionado con respecto al salario que reciben a cambio. No es casual que sus expectativas no sean nunca tomadas en consideración a la hora de definir los horarios de trabajo : gracias a la disponibilidad temporal de los asalariados (y sobre todo de las mujeres) las empresas pueden responder a las exigencias de los clientes, quienes no parecen preocuparse por las condiciones de vida y trabajo de una mano de obra adscrita formalmente a otra empresa. Finalmente, no es sino a través de la renuncia a un turno $u$ otro de trabajo -y de la parte del salario correspondiente- que las mujeres logran limitar las tensiones entre su compromiso laboral y las exigencias de la vida privada, resolviendo, en definitiva, lo que Grossin (1996) denominaría su "ecuación temporal personal". Los cálculos pragmáticos a los que se ven obligados los asalariados del sector de la limpieza para buscar cierto equilibrio entre salario y calidad de vida, constituyen una de las posibles explicaciones de la extensión y feminización de los empleos a tiempo parcial, empleos inicialmente prescritos por la organización del trabajo adoptada de manera estructural en los centros de trabajo.

\subsection{Reordenaciones posibles}

Sin embargo, determinadas reordenaciones del horario de trabajo, bastante modestas de hecho, parecen capaces de modificar profundamente la relación con el empleo, favoreciendo un mayor equilibrio entre la vida laboral y la vida privada. En este sentido, la evaluación de un proyecto piloto de reorganización del trabajo de limpieza en una administración pública ha permitido poner en evidencia la importancia que tienen los horarios de trabajo en la valoración general que hacen los asalariados de los cambios organizativos (Krzeslo \& Lebeer, 2010). La nueva organización puesta en marcha en esta administración permitía a los trabajadores finalizar su trabajo a las 18 : 00 en lugar de a las $20: 00$, reduciendo en 2 horas la duración de la interrupción entre la prestación de servicio efectuada por la mañana (de $6: 00$ a $10: 00$ ) y la de la tarde (de 15 : 00 a $18: 00$ en lugar de $17: 00$ a $20: 00$ ). Según los testimonios recogidos, este horario más concentrado fue acogido positivamente por la mayoría de los trabajadores del sector de la limpieza, al tiempo que el incremento en la regularidad de los contactos con los funcionarios resultó, en términos generales, satisfactorio.

Por otro lado, desde el punto de vista de la organización de las tareas, la racionalización del trabajo consistió en especializar al personal en función del tipo de espacio asignado (despachos, pasillos, cuartos de baño, etc.), con el objetivo de reducir el equipamiento necesario y de favorecer la adquisición de rutinas de trabajo. La valoración de dicha racionalización del trabajo fue bastante similar en todas las partes implicadas. De hecho, se demostró inmediatamente que la valoración de los trabajadores sobre la reorganización de tareas efectuada dependía por completo de su valoración de los horarios de trabajo. De hecho, la satisfacción con respecto a los horarios de trabajo puede también ocultar su valoración de los contratiempos del trabajo. Dicho de otro modo, en un sector como el de la limpieza caracterizado por su flexibilidad, el horario de trabajo constituye el punto de referencia fundamental con el que valorar las condiciones de trabajo. Así, son los horarios de trabajo los que determinan simultáneamente el salario, la posibilidad de mantener el empleo y el disfrute de un tiempo privado dedicado a la vida social y familiar. 


\subsection{La disponibilidad temporal como atributo de la cualificación}

El caso del sector de la limpieza es revelador de un funcionamiento general del mercado de trabajo en el que, como ha señalado Alaluf (2000), más que la cualificación es la confrontación entre disponibilidades temporales -las exigidas por la organización y las ofertadas por el asalariado- lo que resulta determinante en los procesos de vinculación de personas a empleos. Nuestro estudio ha mostrado que, la mayoría de las veces, los trabajadores del sector de la limpieza tratan de conciliar los tiempos de trabajo con las obligaciones de la vida privada mediante la modulación de su implicación en el trabajo. El trabajo a tiempo parcial no evita padecer los inconvenientes de los horarios desincronizados. En la medida en que las guarderías no suelen estar disponibles tan temprano por la mañana o hasta tan tarde por la noche, este tipo de horarios requieren la colaboración del cónyuge, de la familia extendida, o incluso de los vecinos. Por otro lado, desde una perspectiva a más largo plazo, la interrupción de la carrera profesional rara vez es considerada ya que dichas medidas suelen estar destinadas por lo general a los trabajadores ocupados a tiempo completo. Por más que se instauraron precisamente con el objetivo de favorecer la conciliación entre la vida laboral y la vida privada, para muchas trabajadoras de la limpieza este tipo de soluciones simplemente no existe. En definitiva, como ha demostrado el caso belga, la disponibilidad temporal, en ausencia de toda forma de cualificación legalmente validada, constituye para los asalariados del sector la única cualificación que pueden hacer valer para acceder y mantener un empleo, salvaguardando así su salario.

41 Previamente hemos señalado que determinados trabajadores -sobretodo mujerespueden verse obligados a renunciar a alguna de las franjas horarias de la jornada partida como consecuencia de la organización temporal del trabajo y de su incompatibilidad con sus responsabilidades domésticas y familiares. Esta renuncia, sin embargo, no siempre resulta sencilla ya que -junto a la negativa a efectuar horas extra escudándose en los excesivos tiempos de desplazamiento requeridos, en problemas con los horarios o en la ausencia de un salario extra [5] - es a menudo interpretada por los superiores como un "rechazo al trabajo" merecedor de sanciones. La exigencia de una disponibilidad extrema - al igual que la implementación de una disciplina de trabajo- se sostiene bajo la amenaza de la pérdida del empleo.

\section{Conclusiones}

42 En el sector de la limpieza la organización temporal de las tareas determina en gran medida el conjunto de las condiciones de trabajo del personal, así como el horizonte temporal del empleo y la reordenación del tiempo de trabajo. Los empleos razonables desde el punto de vista del régimen de trabajo y del horario no abundan. Tanto hombres como mujeres están sometidos a las dificultades derivadas de la jornada partida que divide el tiempo de trabajo en bloques homogéneos (habitualmente uno de mañana y otro de tarde). En este contexto, los asalariados se ven confrontados a un dilema permanente entre la necesidad económica de lograr más horas de trabajo y la búsqueda de una coordinación entre los distintos tiempos sociales. Las mujeres, en particular, padecen simultáneamente el trabajo a tiempo parcial -sobre cuya duración no suelen poder negociar- y los horarios de trabajo atípicos. Se constata así que las expectativas temporales de los asalariados no son tomadas en consideración sino a 
través del filtro distorsionador de una representación sexuada y tradicional de los roles que se traduce en una división sexual del trabajo y del empleo.

Uno de los principales desafíos para la mejora de las condiciones de trabajo en el sector de la limpieza radica en el control de los horarios de trabajo. En un contexto en el que proliferan los discursos que reivindican la individualización de los tiempos de trabajo en nombre de su mejor conciliación con la vida privada, es fundamental abordar la cuestión del espacio cada vez mayor que ocupa el tiempo de trabajo en el tiempo de vida de las personas. Los tiempos de trabajo adscritos a los asalariados del sector de la limpieza reflejan los determinantes de la posición social que les es asignada. Dicha posición social tendría un primer factor determinante en la condición de sujetos subalternos de la empresa que tienen dichos trabajadores, en tanto en cuanto sus horarios de trabajo se encuentran subordinados a los horarios del resto de asalariados de la empresa, es decir, de los empleados de la empresa-cliente. Estaríamos ante una subordinación que remite a sus condiciones de empleo, a su estatuto y a la consideración atribuida a su trabajo.

En un contexto marcado por una exigencia de disponibilidad temporal cada vez mayor, otro determinante de su posición social es el modo en que el tiempo de trabajo es distribuido en los distintos centros de trabajo y, consecuentemente, el salario al que dará derecho. A este respecto, la diferenciación sexual del trabajo resulta fundamental. Más concretamente, el reparto de los horarios de trabajo que hemos podido constatar en nuestra investigación se basa en un rosario de ideas preconcebidas a propósito de la supuesta contraposición entre cualidades masculinas y femeninas. Estas ideas preconcebidas servirían para justificar la atribución a los hombres de jornadas de trabajo a tiempo completo, las tareas de limpieza industrial y el manejo de maquinaria y a las mujeres la jornada de trabajo a tiempo parcial y la limpieza de los despachos.

El tercer elemento determinante de la posición social de estos trabajadores (que colocaría la cuestión de los horarios en el centro del debate sobre las condiciones de trabajo en el sector) sería la escasa importancia acordada al impacto de dichos horarios en la articulación entre tiempos de trabajo y tiempos privados. Para un buen número de mujeres, la falta de coordinación entre los diferentes tiempos sociales es algo bien tangible. Las dificultades planteadas por el cuidado de los hijos no pueden resolverse únicamente mediante la movilización de la familia o de los vecinos, sino que requerirá a menudo la renuncia a una parte del tiempo de trabajo y, por lo tanto, a una parte del salario que se antoja, no obstante, imprescindible para poder escapar de la condición de "trabajador pobre".

Frente al riesgo de una legitimación de la división sexual del trabajo por medio de las relaciones de género existentes en el seno de la familia conviene, no obstante, enfatizar el carácter contingente de la organización del trabajo. Centrar demasiado la atención en los factores familiares que motivan la elección del trabajo a tiempo parcial y la renuncia a una parte del trabajo por parte de determinadas trabajadoras, supone olvidar que la perspectiva de la "conciliación" busca cuestionar también los modos de gestión de la mano de obra (Alonzo, Angeloff \& Maruani, 2005). A diferencia, sin embargo, de lo que ocurre en otros grupos profesionales feminizados como las enfermeras -que gozan de cierta estabilidad en el empleo y que, a pesar de las limitaciones objetivas de la actividad, pueden hacer valer hasta cierto punto, individual y colectivamente, las obligaciones de la vida familiar para lograr cierta reorganización de los tiempos de trabajo (Bouffartigue \& Bouteiller, 2006)- en el caso de las 
trabajadoras precarias de la limpieza este tipo de dimensiones no son tomadas en consideración a pesar de que el recurso a la jornada partida y a los horarios desincronizados no tengan nada de ineluctables.

\section{BIBLIOGRAFÍA}

Alaluf, M. (2000). Le travail du temps. In Gilbert de Terssac \& Diane-Gabrielle Tremblay (Dirs.), Oú va le temps de travail? (pp. 203-213). Toulouse : Octarés Éditions.

Alonzo, P., Angeloff, T. \& Maruani, M. (2005). Travail, famille et genre : une relation à double sens. In Margaret Maruani (Dir.), Femmes, genre et sociétés. L'état des savoirs (pp. 372-380). Paris : La Découverte.

Angeloff, T. (1999). Des miettes d'emploi : temps partiel et pauvreté. Travail, Genre et Sociétés, 1 , 43-70.

Barrère-Maurisson, M. A. (2000). Partage des tâches, des temps dans les ménages et parité professionnelle. In Evelyne Istace, Michel Laffut, Robert Plasman \& Christine Ruyters (Eds.), Sphères privée et professionnelle. Vers une recomposition des rôles et des actions (pp. 295-311). Bruselas : De Boeck.

Bouffartigue P., \& Bouteiller, J. (2006). Jongleuses en blouse blanche. Temporalités, 4. Retirado en Mayo, 5, 2012 de http://temporalites.revues.org/344.

Brochiez, C. (2001). Des jeunes corvéables. L'organisation du travail et la gestion du personnel dans un fast-food. Actes de la recherche en sciences sociales, 138, 73-83.

Cortese, V., Martínez, E., \& Munar, L. (2003, Noviembre). Organisation professionnelle et mobilisation de la main-d'œuvre dans la sous-traitance de services. Le cas de la sécurité privée. Comunicación presentada en las IXèmes Journées de Sociologie du Travail. Paris, Francia.

European Federation of Cleaning Industries (EFCI) (2006). The Cleaning Industry in Europe. Bruxelles : European Federation of Cleaning Industries.

Grossin, W. (1996). Pour une science du temps. Introduction à l'écologie temporelle. Toulouse : Octarès.

Krzeslo, E., \& Lebeer, G. (2010). La perception des changements organisationnels et horaires du chantier du Service Public Fédéral des Finances par le personnel de nettoyage. Enquête auprès des salariés de la firme Euroclean. Informe de investigación realizado a petición Centre de Formation du Nettoyage. METICES - Université Libre de Bruxelles. Bruselas : Bélgica.

Lebeer, G., De Schampheleire, J., \& Krzeslo, E. (2009). Temps de travail, charge de travail et conciliation entre la vie privée et la vie professionnelle. Informe de investigación realizado a petición Centre de Formation du Nettoyage. METICES - Université Libre de Bruxelles. Bruselas : Bélgica.

Martínez, E. (2010). Les salariés à l'épreuve de la flexibilité. Bruselas : Presses de l'Université de Bruxelles.

Munar, L., \& Lebeer, G. (2005). Nature et contenus de la relation de sous-traitance dans le secteur du nettoyage. Travail Emploi Formation, 6, 6-31. 
Puech, I. (2004). Le temps du remue-ménage. Conditions d'emploi et de travail de femmes de chambre. Sociologie du travail, 46, 150-167.

Scandella, F. (2010). Les dessous d'une industrie en pleine expansion. HesaMag, 2, 13-16.

Thoemmes, J. (2000). Vers la fin du temps de travail ? París : PUF.

\section{NOTAS}

1. Empleamos el término de "limpieza de oficinas" para diferenciar éste tipo de actividades del servicio de limpieza en instalaciones industriales, la recogida de basuras y la limpieza de cristales. El servicio de limpieza de oficinas incluiría el espacio de los despachos, así como otros espacios colectivos equiparados (salas de reunión, espacios de paso, comedores, servicios...)

2. La tasa de penetración mide la cobertura por parte del mercado de un producto o servicio. Es el resultado de dividir la demanda actual de un producto o servicio por la demanda potencial del mismo.

3. En el sector de la limpieza, la empresa "entrante" que se hace con el contrato del servicio está obligada a mantener al personal previamente ocupado en el centro, garantizándoles el empleo al menos por un período de seis meses. La continuidad de la relación de trabajo en la empresacliente prima pues sobre el contrato de trabajo con el empleador. Los trabajadores permanecen en el mismo centro de trabajo utilizando, llegado el caso, el uniforme de las sucesivas empresas adjudicatarias del servicio.

4. En Bélgica, mientras que las situaciones de monoparentalidad afectaban al $8,4 \%$ del conjunto de los asalariados (5,6\% en el caso de los hombres, $11,7 \%$ entre las mujeres), dicho porcentaje se elevaba al $11,6 \%$ en el sector de la limpieza (5,5\% entre los hombres, $14,8 \%$ entre las mujeres). Como muestran estos datos, la disparidad proviene, básicamente, de la situación de las mujeres (Fuente: Base de datos de la Banque Carrefour de la Sécurité Sociale, 2008).

5. La disponibilidad temporal consistente en la aceptación de un trabajo complementario o de horarios incómodos no está reconocida, ni compensada mediante el pago de un salario extra. El marco normativo institucionalizado a nivel de rama de actividad refleja claramente, a escala de la jornada laboral, esta exigencia de disponibilidad: situando en las 22:00 el umbral a partir del cual el trabajo nocturno debe ser compensado por medio de un salario extra (cuando en la reglamentación belga del tiempo de trabajo, el trabajo nocturno hace referencia a las prestaciones laborales efectuadas entre las 20:00 y las 6:00), no respetando los límites mínimos de duración de la jornada de trabajo e ignorando los inconvenientes derivados de la jornada partida.

\section{RESÚMENES}

A partir de los resultados de un estudio cualitativo efectuado en distintos centros de trabajo belgas, este artículo muestra la importancia que tienen las desigualdades de género dentro del sector de la limpieza, analizando al mismo tiempo su dinámica específica en el ámbito de los regímenes de trabajo, de las modalidades concretas de organización de tareas y de la gestión de la mano de obra. El artículo pone en evidencia que el empleo de mujeres en el sector de la limpieza refleja los efectos de una doble segregación. En primer lugar, la segregación derivada de las 
condiciones específicas de subordinación que caracterizan al trabajo de limpieza efectuado - cada vez más - en el marco de relaciones de subcontratación. En segundo lugar, aquella que se desprende de las tensiones, padecidas especialmente por las mujeres, entre regímenes y horarios de trabajo no negociables - trabajo a tiempo parcial no elegido voluntariamente, jornadas partidas de trabajo y con horarios desincronizados - y obligaciones de la vida privada.

A partir dos resultados de um estudo qualitativo conduzido em diversos locais de actuação de empresas do sector das limpezas na Bélgica, este artigo mostra a importância das desigualdades de género no sector e decompõe os mecanismos inerentes aos regimes de trabalho e às modalidades específicas de organização do trabalho e de gestão da mão-de-obra. Este estudo coloca também em evidência o facto de o emprego das mulheres neste tipo de actividade registar os efeitos de uma dupla segregação : antes de mais, uma segregação que se reporta às condições particulares de subordinação que caracterizam o trabalho de limpeza, cada vez mais exercido no quadro de relações de subcontratação e, depois, uma segregação decorrente de tensões que afectam mais particularmente as mulheres, entre regimes e horários de trabalho não negociáveis - trabalho a tempo parcial imposto e horários segmentados e dessincronizados - e os constrangimentos da vida privada.

$\mathrm{Au}$ départ des résultats d'une étude qualitative menée dans plusieurs chantiers de nettoyage en Belgique, cet article montre l'importance des inégalités de genre dans le secteur et en décompose les mécanismes, propres aux régimes de travail et aux modalités spécifiques de l'organisation du travail et du management de la main d'œuvre. Il met en évidence que l'emploi des femmes dans le nettoyage enregistre les effets d'une double ségrégation: tout d'abord, celle due aux conditions particulières de subordination qui caractérisent le travail du nettoyage exercé de plus en plus souvent dans le cadre de relations de sous-traitance et ensuite celle découlant des tensions que subissent plus particulièrement les femmes entre des régimes et des horaires de travail non négociables - temps partiel non choisi et horaires coupés et décalés - et les contraintes de la vie privée.

From the results of a qualitative study conducted in several sites of cleaning in Belgium, this article shows the importance of gender inequalities in the sector and decomposes the mechanisms, related to the specific work regimes and to the specific modalities of work organization and management. It brings to light that women's employment in cleaning records the effects of a double segregation: first of all, that due to the particular conditions of subordination which characterize the work of the cleaning exercised more and more often within the framework of relations of subcontracting and then that ensuing from the tensions which undergo more particularly the women between not negotiable work regimes and working schedules - not chosen part-time and cut and shifted schedules - and the constraints of the private life.

\section{ÍNDICE}

Palabras claves: sociología del trabajo, sector de la limpieza, condiciones de trabajo, relaciones de género

Keywords: sociology of work, cleaning sector, working conditions, gender relations

Palavras-chave: sociologia do trabalho, sector das limpezas, condições de trabalho, relações de género

Mots-clés: sociologie du travail, secteur du nettoyage, conditions de travail, relations de genre 


\section{AUTORES}

\section{GUY LEBEER}

METICES - Université Libre de Bruxelles, 44 avenue Jeanne, 1050 Bruxelles - Belgique glebeer@ulb.ac.be

\section{ESTEBAN MARTINEZ}

METICES - Université Libre de Bruxelles, 44 avenue Jeanne, 1050 Bruxelles - Belgique emartin@ulb.ac.be 